\title{
Nonlinear Switching Behaviors in a Compact All-Semiconductor Optical-Amplifier Sagnac Interferometer Device
}

\author{
Jiun-Haw Lee, Ding-An Wang, Yean-Woei Kiang, Hsin-Jiun Chiang, \\ Ding-Wei Huang, and C. C. Yang, Senior Member, IEEE
}

\begin{abstract}
Nonlinear switching effects in a GaAs-AlGaAs allsemiconductor optical-amplifier loop device with a multimode interference waveguide amplifier (MMIWA) for closing the loop was investigated experimentally and numerically. The miniaturized device, designed to imitate a nonlinear optical loop mirror (NOLM), has a latency more than one hundred times smaller than that of a NOLM. Also, because it used an MMIWA for replacing a coupler in a conventional NOLM, its operation was quite different from that previously reported. In CW signal operation, the nonlinear switching behavior resulted from the combined effect of nonlinear coupling in the MMIWA and the amplification and lateral field redistribution of the signal through the loop structure. Efficient self-switching and cross-polarized switching were observed. Numerical simulations showed consistent trends in varying device parameters.
\end{abstract}

Index Terms - Gain saturation, nonlinear optical loop mirror, optical switch, semiconductor optical amplifier.

\section{INTRODUCTION}

A LL-OPTICAL switches are promising devices for highspeed signal processing and communications, particularly in wavelength-division multiplexing (WDM) and time-division multiplexing (TDM) systems. Among those all-optical devices, the nonlinear optical loop mirror (NOLM) or nonlinear Sagnac interferometer is one of the most widely used [1]-[3]. Basically, a NOLM consists of an optical fiber loop connected with a fiber coupler [4], [5]. Counterpropagating optical signals of unequal intensities acquire different nonlinear phase shifts in the loop, leading to nonlinear switching after they recombine at the fiber coupler. The required optical nonlinearity comes from the Kerr effect in the fiber. Compared with other all-optical devices, a NOLM has the advantages of inherent stability (because of its Sagnac interferometric arrangement) and speed (because of the use of the Kerr effect). For increasing the asymmetry of the two counterpropagating signals, a fiber amplifier has been asymmetrically inserted into the loop. Such a device is usually called a nonlinear amplifying loop mirror

Manuscript received April 5, 1999; revised May 25, 1999. This research was supported by National Science Council, The Republic of China, under Grant NSC 87-2215-E002-006, Grant NSC 87-2215-E002-007, Grant NSC 87-2112-M-002-025 and Grant NSC 88-2215-E-002-016.

The authors are with the Department of Electrical Engineering and the Graduate Institute of Electro-Optical Engineering, National Taiwan University, Taipei, Taiwan, R.O.C.

Publisher Item Identifier S 0018-9197(99)06795-0.
(NALM). A NOLM or NALM needs a long fiber loop for accumulating optical nonlinearity. Typically, a fiber loop length of several hundred meters is required [6], [7], corresponding to a device latency on the order of 500 ns. Meanwhile, the relatively weak Kerr effect makes the switching power quite high (typically, 1-W peak power).

To improve the operation of the device, a semiconductor optical amplifier (SOA) has been asymmetrically inserted into the fiber loop [8]. In such a device, nonlinear switching relies on gain saturation and an associated phase change in the SOA. Because such an optical nonlinearity is much more effective than the Kerr effect, the switching power can be greatly reduced. Meanwhile, since the fiber loop is not used for optical nonlinearity, its length can be decreased to $0.5 \mathrm{~m}$ or less. Such a device, with a control pulse to generate the asymmetry of the two counterpropagating signals in the loop, has been used for terahertz optical demultiplexing [9]. The use of an SOA has much improved the device operation. However, a fiber loop, several tenths of a meter in length, still corresponds to a latency of around $2 \mathrm{~ns}$. Such a long latency may reduce the processing speed in using a conventional NOLM. Besides, its bulky configuration makes the integration difficult.

To make the device more compact, the fabrication of a monolithically integrated nonlinear Sagnac interferometer was reported [10]. In this all-semiconductor NOLM, an SOA is inserted into a passive waveguide loop with a multimode interference waveguide amplifier (MMIWA), serving as a 3-dB coupler, to close the loop. The latency of such a NOLM has been tremendously reduced. It also shows the advantages of compactness, low cost, and mass production. However, the required regrowth technique for defining the active and passive sections increases the difficulty of device fabrication. In this paper, we propose an all-semiconductor optical-amplifier miniature device based on the Sagnac interferometer configuration. It is monolithically made of a GaAs-AlGaAs SOA. Similar to that in [10], an MMIWA is used to replace the conventional fiber coupler for closing the loop. The latency of such a device is much reduced because of its small size. For instance, the device to be described in the following has a loop length of only $2.888 \mathrm{~mm}$ (including the MMIWA), corresponding to a latency of only $31.8 \mathrm{ps}$. The proposed device is similar in configuration to the NOLMtype devices previously discussed. However, its operation 


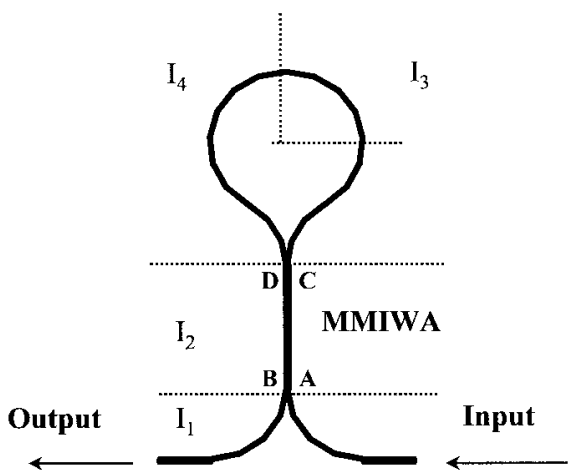

Fig. 1. Layout of the all-semiconductor optical-amplifier Sagnac interferometer device. The electrode is divided into four disconnected sections denoted by $I_{1}-I_{4}$

mechanism is quite different. It depends not only on the optical nonlinearity in the loop structure but also on the nonlinear coupling effect in the MMIWA. Experimentally, with CW signals we observed quite efficient self-switching and alloptical modulation. Numerical simulations showed consistent trends and proved the important role of either the loop structure or MMIWA in nonlinear switching.

In Section II, the design and fabrication processes of the device are described. Experimental procedures and results are discussed in Section III. Then, a theoretical model is developed to simulate the operation of the device in Section IV. Finally, conclusions are given in Section V.

\section{Design And Fabrication Process}

Fig. 1 shows the layout of the device. The device consists of four parts: the loop, the MMIWA, the input port, and the output port. All parts were made of GaAs-AlGaAs ridgewaveguide SOA's. The loop has a radius of $300 \mu \mathrm{m}$ with a ridge width of $4 \mu \mathrm{m}$ (expected to form a single-lateralmode waveguide). The loop is connected to an MMIWA with a length of $480 \mu \mathrm{m}$ and a ridge width of $8 \mu \mathrm{m}$. The input and output ports also have a ridge width of $4 \mu \mathrm{m}$. Both have lengths of about $600 \mu \mathrm{m}$. The ridge waveguides were fabricated with the cryo-etching technique [11], [12]. The etching depth was $1.8 \mu \mathrm{m}$. For injecting different currents in different areas, we divided the electro-pad into four disconnected regions, indicated by the injection currents $I_{1}$ (input-output region), $I_{2}$ (MMIWA region), $I_{3}$ (one-quarter of the loop), and $I_{4}$ (threequarters of the loop). $I_{3}$ and $I_{4}$ were designed to be disconnected for the purpose of future research. Such a device was fabricated on a four-period GaAs-AlGaAs multiple-quantumwell epitaxial structure. The GaAs wells (6.5 nm thick) and $\mathrm{Al}_{0.25} \mathrm{Ga}_{0.75}$ As barriers $(20 \mathrm{~nm})$ were sandwiched by two 140-nm-thick i- $\mathrm{Al}_{0.25} \mathrm{Ga}_{0.75} \mathrm{As}$ separate confinement layers, and $\mathrm{p}-\mathrm{Al}_{0.6} \mathrm{Ga}_{0.4} \mathrm{As}$ and $n-\mathrm{Al}_{0.6} \mathrm{Ga}_{0.4}$ As cladding layers, both $1.5 \mu \mathrm{m}$ in thickness. On the top, a $50-\mathrm{nm} \mathrm{p}^{+}-\mathrm{GaAs}$ layer was grown for good ohmic contact. The electroluminesence (EL) peaks of the TE and TM polarization of the epitaxial structure were at 849.6 and $844.8 \mathrm{~nm}$, respectively. The fabrication procedures were the same as those of typical semiconductor lasers except the etching technique for forming ridge waveguides. To achieve vertical and smooth etched walls for reducing bending loss and to minimize surface damage, we used the ultraviolet(UV)-assisted cryo-etching technique [11], [12]. This technique utilizes a UV laser to define the etching feature at a low temperature. Compared to the typical chemical wet etching techniques, it can provide fine etching features. Compared to the reactive ion etching techniques, it leads to little damage to the etched surface [13].

In the all-SOA device described above, gain saturation is the mechanism used for nonlinear switching. With different injection current densities onto the electro-pads of $I_{3}$ and $I_{4}$, the asymmetrically distributed gain and gain saturation for the counterpropagating signals in the loop make its function similar to that of a NOLM when pulsed signals are used. In other words, the absolute difference in intensity of the two counterpropagating signals controls the relative differences in intensity and phase of the two signals after loop circulation. However, for $\mathrm{CW}$ or quasi-CW signals, the asymmetry is unimportant for power-dependent switching. Note that a major difference between our device and a conventional NOLM is the coupler used to close the loop. In a conventional device, the coupler is a linear-optical device. It simply splits input power at a fixed ratio. However, in our device, an MMIWA is used which can provide the function of nonlinear coupling [14]. In other words, the lateral distribution of optical intensity of the MMIWA and, hence, the power distribution of the counterpropagating signals coupled into the loop depend on the input power level. Therefore, the MMIWA plays an important role in nonlinear switching of the whole device. Although the function of the loop with CW or quasi-CW signals is simply to amplify the signals with the input ratio, it also plays an important role in the operation of the whole device. First, the amplification of the signals is important for enhancing the nonlinear coupling in the MMIWA. Second, the loop configuration redistributes the lateral intensity profile of the MMIWA. As will be seen in the following experimental and numerical results, the redistribution is important for the nonlinear switching operation of the whole device. Therefore, our new device is different from a conventional NOLM because of the combined effects of the loop and coupler. Although only the case of a CW signal will be discussed in this paper, it is expected that the combination of the loop and coupler functions in our device should lead to larger varieties of device operation, compared with the conventional devices, when pulsed signals are used. Hence, in operation of the device, the injection current $I_{3}+I_{4}$ must be sufficiently large for providing gain to compensate for the bending loss of the curved waveguide and to amplify the counterpropagating signals for efficient nonlinear coupling in the MMIWA. Meanwhile, the $I_{2}$ value should be appropriate for producing significant gain saturation in the MMIWA so that nonlinear coupling there can be effective. Regarding $I_{1}$, it amplifies the input and output signals.

\section{EXPERIMENTAL PROCEDURES AND RESUlTS}

\section{A. Basic Operation of the Device}

As shown in Fig. 2, in the experiments we used a CW tunable Ti : sapphire laser with various power levels as input 


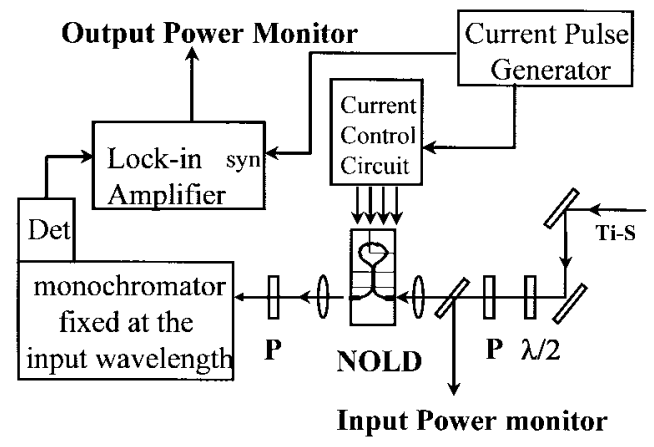

Fig. 2. Experimental setup for the measurement of the device performance. The notations Ti-S, $\lambda / 2, P$, and Det represent the Ti:sapphire laser, half-wave plate, polarizer, and detector, respectively.

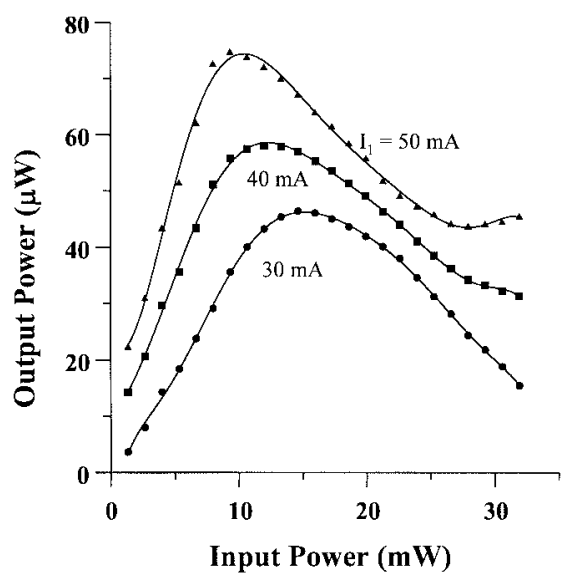

Fig. 3. Output power as a function of input power with different $I_{1}$ levels. $I_{2}$ is fixed at $55 \mathrm{~mA}$ and $I_{3}+I_{4}$ is set at $300 \mathrm{~mA}$. The optical signal is TE-polarized and is at $849.6 \mathrm{~nm}$.

signals and monitored the power variation from the output port at the same wavelength. It was estimated that the power coupled into the input-port waveguide was about $1 \%$ of the input power. For reducing the output noise, the output signal was passed through a monochromator allowing a bandwidth of only $2 \mathrm{~nm}$ before a photodetector. Meanwhile, a polarizer was used for selecting the output polarization. Fig. 3 shows three sets of data demonstrating efficient power-dependent switching. The horizontal axis represents the input-port power before entering the input waveguide. The continuous curves fitting the data points are meant to guide the eyes (true for Figs. 3-11). In this measurement, $I_{2}=55 \mathrm{~mA}, I_{3}+I_{4}=300 \mathrm{~mA}$, and $I_{1}$ was varied from 30 to $50 \mathrm{~mA}$, as shown in the figure. The input signal was TE polarized and the wavelength was at $849.6 \mathrm{~nm}$, which was at the TE gain peak. These curves show the depression of output power when the input power reaches a certain value. They show the typical powerdependent phenomenon observed in a NOLM. With increasing $I_{1}$, the peak value of the output power becomes higher. Meanwhile, the input power for the output peak, which can be used to represent the switching power, decreases with increasing $I_{1}$. The reason for this trend is quite clear that the real input power entering the MMIWA was increased with $I_{1}$. Meanwhile, the output power was amplified with the

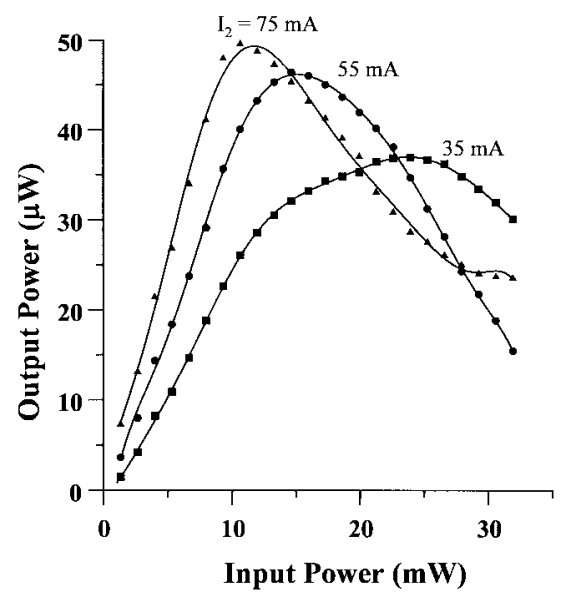

Fig. 4. Output power as a function of input power with different $I_{2}$ levels. $I_{1}$ is fixed at $30 \mathrm{~mA}$ and $I_{3}+I_{4}$ is set at $300 \mathrm{~mA}$. The optical signal is TE-polarized and is at $849.6 \mathrm{~nm}$.

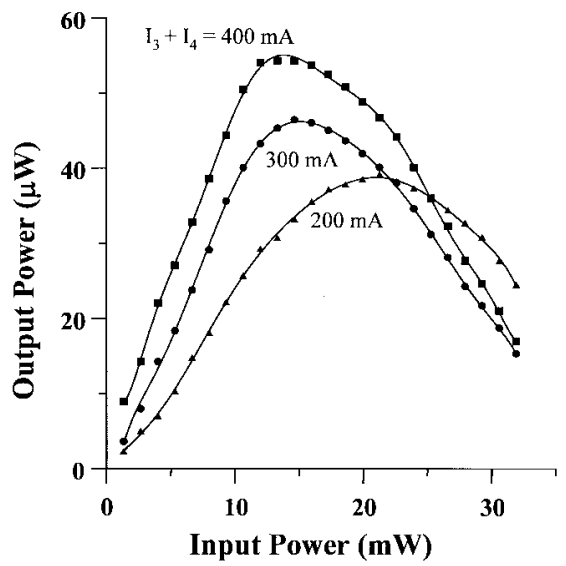

Fig. 5. Output power as a function of input power with different $I_{3}+I_{4}$ levels. $I_{1}$ is fixed $30 \mathrm{~mA}$ and $I_{2}$ is set at $55 \mathrm{~mA}$. The optical signal is TE-polarized and is at $849.6 \mathrm{~nm}$.

injection of $I_{1}$. In Fig. 4, again with TE-polarized signals at $849.6 \mathrm{~nm}$, we vary the $I_{2}$ value from 35 to $75 \mathrm{~mA}$, while $I_{1}$ and $I_{3}+I_{4}$ are kept at 30 and $300 \mathrm{~mA}$, respectively. Here, we can also observe that the switching power decreases with increasing $I_{2}$. Meanwhile, the output peak value increases with $I_{2}$. In addition, the output peak becomes sharper as $I_{2}$ increases. These trends imply that within a certain range a larger injection current onto the MMIWA leads to a more efficient nonlinear switching result. Such a conclusion can be easily understood because a larger $I_{2}$ results in stronger gain saturation and, hence, more efficient nonlinear coupling in the MMIWA. Then, in Fig. 5, we fix $I_{1}$ at $30 \mathrm{~mA}, I_{2}$ at $55 \mathrm{~mA}$, and vary $I_{3}+I_{4}$ from 200 to $400 \mathrm{~mA}$. Again, the switching power decreases and the output peak level increases with increasing $I_{3}+I_{4}$. One can see that the gain in the loop is quite important for efficient nonlinear switching. It is noted that the relative changes in the three curves of Figs. 4 and 5 are similar. However, the injection current increments of the two figures are quite different. In terms of current density, the increments for $I_{2}$ (Fig. 4) and $I_{3}+I_{4}$ (Fig. 5) are 0.521 and $1.326 \mathrm{kA} / \mathrm{cm}^{2}$, respectively. We can see that the nonlinear 


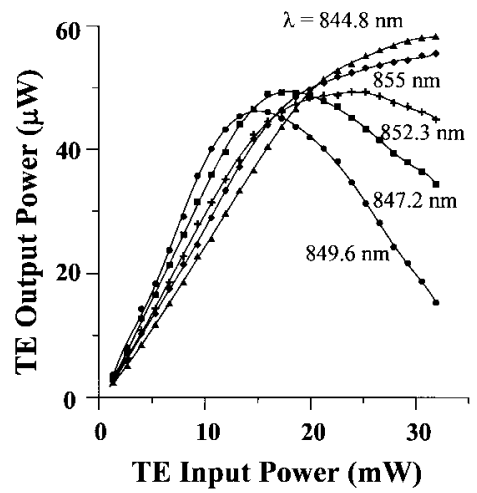

Fig. 6. Output power as a function of input power with the wavelengths at $844.8,847.2,849.6,852.3$, and $855 \mathrm{~nm} . I_{1}, I_{2}$, and $I_{3}+I_{4}$ are set at 30,55 , and $300 \mathrm{~mA}$, respectively. The optical signal is TE polarized.

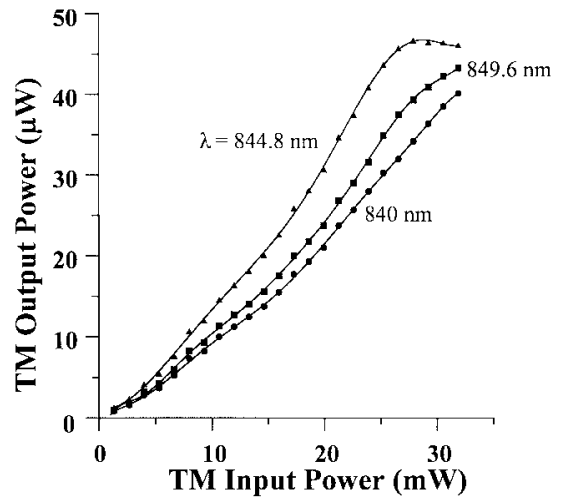

Fig. 7. Output power as a function of input power with the wavelengths at $840,844.8$, and $849.6 \mathrm{~nm} . I_{1}, I_{2}$, and $I_{3}+I_{4}$ are set at 30,55 , and $300 \mathrm{~mA}$, respectively. The optical signal is TM polarized.

switching result is more sensitive to the variation of the gain constant in the MMIWA than in the loop.

\section{B. Wavelength and Polarization Dependencies of the Device Operation}

In Fig. 6, we vary the input signal wavelength from 844.8 to $855 \mathrm{~nm}$ with five different values centered at $849.6 \mathrm{~nm}$, which corresponds to the TE gain peak. In these measurements, $I_{1}$ was $30 \mathrm{~mA}, I_{2}$ was $55 \mathrm{~mA}$, and $I_{3}+I_{4}$ was $300 \mathrm{~mA}$. The input signals were TE polarized. We can see that the switching power is the smallest and the nonlinear switching is most efficient at the gain peak. As the gain value becomes smaller on both sides of $849.6 \mathrm{~nm}$, gain saturation and, hence, the nonlinear switching effect become weaker. We also conducted a similar experiment with TM-polarized input signals under the same injection current conditions. Fig. 7 shows the results of three wavelengths centered at $844.8 \mathrm{~nm}$, which corresponds to the TM gain peak. In these cases, no significant nonlinear switching behaviors were observed in the concerned input power range. This is probably because the gain and gain saturation are weaker for the TM signals in our sample. From the results in Fig. 6, one can see that device operation is quite sensitive to wavelength. Hence, it has the potential for WDM applications. However, such an application relies on an understanding of multiwavelength operation of such a device.

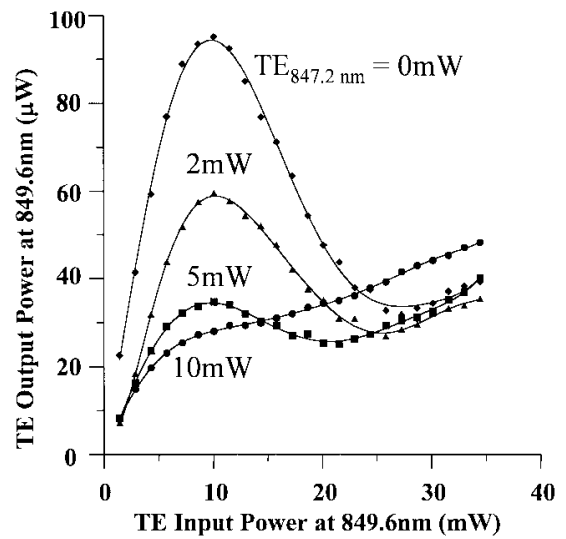

Fig. 8. Signal output power at $849.6 \mathrm{~nm}$ as a function of input power at the same wavelength when the power of the control laser at $847.2 \mathrm{~nm}$ is varied from 0 to $10 \mathrm{~mW}$. $I_{1}, I_{2}$, and $I_{3}+I_{4}$ are set at 30,55 , and $300 \mathrm{~mA}$, respectively. Both signal and control lasers are TE polarized.

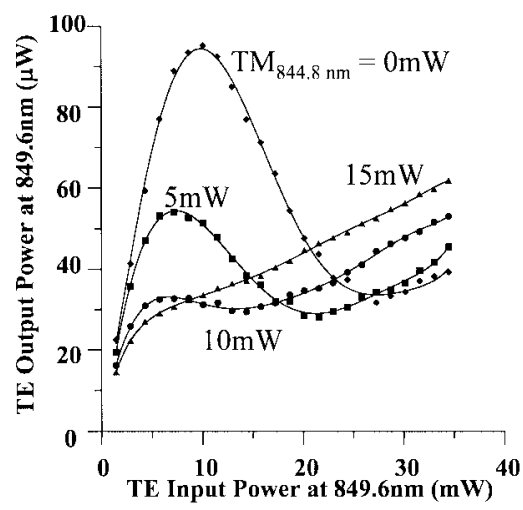

Fig. 9. TE signal output power at $849.6 \mathrm{~nm}$ as a function of input power at the same wavelength and polarization when the power of the TM control laser at $844.8 \mathrm{~nm}$ is varied from 0 to $15 \mathrm{~mW} . I_{1}, I_{2}$, and $I_{3}+I_{4}$ are set at 30,55 , and $300 \mathrm{~mA}$, respectively.

\section{Cross-Switching between Two Signals with Different Wavelengths/Polarization}

For the cross-switching study, we prepared two tunable CW $\mathrm{Ti}$ : sapphire laser, for simultaneous coupling into the input port of the device. In Fig. 8, we show four sets of data with the TE signal at $849.6 \mathrm{~nm}$ affected by the TE control laser at $847.2 \mathrm{~nm}$, which is varied from 0 to $10 \mathrm{~mW}$. The injection current conditions are $I_{1}=30 \mathrm{~mA}, I_{2}=55 \mathrm{~mA}$ and $I_{3}+I_{4}=300 \mathrm{~mA}$. One can see that the control laser reduces the switching power of the signal. However, because the control laser consumes carriers and, hence, decreases the gain of the signal, the contrast ratio for nonlinear switching becomes smaller as the control laser power increases. Then, in Figs. 9 and 10, we consider the cases of cross switching between TE and TM polarization with the wavelengths at their gain peaks, respectively. In Fig. 9, the nonlinear switching of the TE signal is controlled by the TM laser. The similar trends in Figs. 8 and 9 indicate the same physical mechanism that the control laser (in either polarization) can enhance gain saturation and, hence, decrease the switching power. However, the contrast ratio is reduced. This is a typical phenomenon in nonlinear switching. As shown in Fig. 10, although nonlinear switching in TM polarization is less efficient, its behavior 


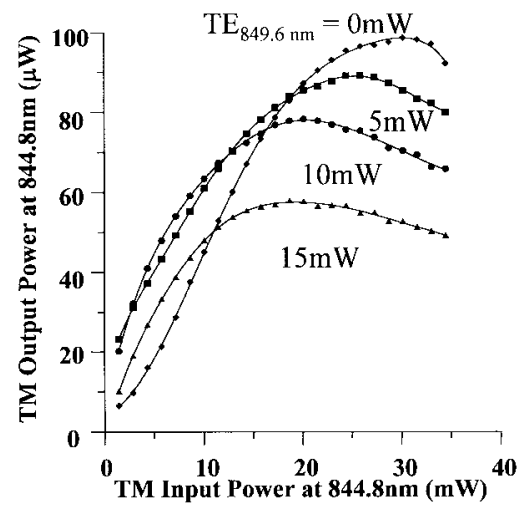

Fig. 10. TM signal output power at $844.8 \mathrm{~nm}$ as a function of input power at the same wavelength and polarization when the power of the TE control laser at $849.6 \mathrm{~nm}$ is varied from 0 to $15 \mathrm{~mW}$. $I_{1}, I_{2}$, and $I_{3}+I_{4}$ are set at 30,55 , and $300 \mathrm{~mA}$, respectively.

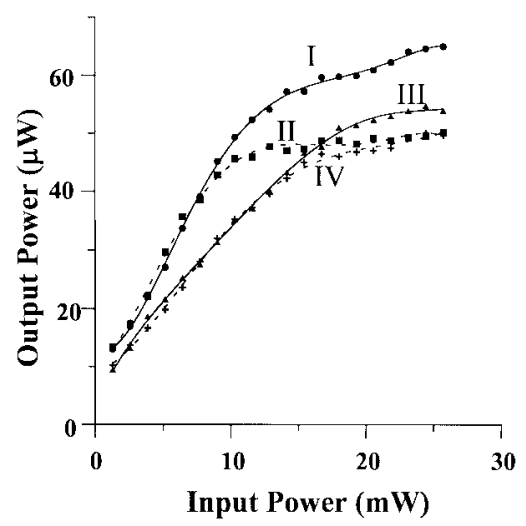

Fig. 11. Comparison between the mirror and loop cases. Solid curves (I and III) and dotted curves (II and IV) represent the mirror and loop cases, respectively. $I_{1}$ is fixed at $300 \mathrm{~mA}$. $I_{2}$ is varied from $200 \mathrm{~mA}$ (III and IV) to $250 \mathrm{~mA}$ (I and II). $I_{3}+I_{4}$ in the loop case is $30 \mathrm{~mA}$. The optical signal at $849.6 \mathrm{~nm}$ is TE polarized.

when affected by a cross-polarized control laser is similar. We have also conducted the cross-polarized switching experiments with the same wavelength. However, the nonlinear switching effect is not as good as those shown in Figs. 9 and 10.

Note that the switching contrast ratio of a conventional NOLM is defined as the power percentage contrast between the reflected and transmitted signals (i.e., power emerging from the input and output ports). However, because we have difficulty in measuring the power emerging from the input port (due to the reflection at the input facet), we cannot obtain the switching ratio from experiments.

\section{The Function of the Loop}

To demonstrate experimentally the function of the loop, we compared the switching results between the loop device and the same device with the loop removed. With the loop removed (called the mirror case), the signal is reflected from the loop end of the MMIWA. In Fig. 11, we demonstrate four sets of data. Solid (curves I and III) and dotted (curves II and IV) curves represent the results of the mirror and loop cases, respectively. For comparison, we fixed $I_{1}$ at $300 \mathrm{~mA}, I_{3}+I_{4}$ at $30 \mathrm{~mA}$ (only for the loop case), and considered two $I_{2}$

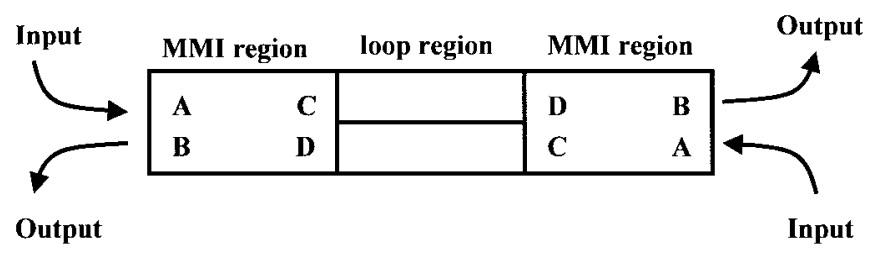

Fig. 12. Model for simulating the device operation.

values at 200 (curves III and IV) and 250 (curves I and II) $\mathrm{mA}$ in both cases. The input signals were TE polarized and the wavelength was fixed at $849.6 \mathrm{~nm}$. In the loop case, $I_{3}+I_{4}$ was adjusted to a smaller value $(30 \mathrm{~mA})$ so that the behaviors in the low-power range are close to those of the mirror case. From the comparison between the solid and dotted curves, one can see that the existence of the loop in the device leads to earlier saturation of the output power as the input power increases. It has been shown earlier in this section that, with a larger $I_{3}+I_{4}$, the device with the loop structure resulted in more efficient nonlinear switching. The results in Fig. 11 demonstrate the importance of the loop structure in the device.

\section{Simulation Results}

To understand the physical mechanisms behind the nonlinear switching, we conducted numerical simulations on the device operation. In either the MMIWA or loop region, we solved a set of equations for the forward- and backwardpropagating wave fields that consumed the same carrier density distribution. For simulations, the device was modeled with the configuration shown in Fig. 12 to account for the counterpropagation of waves. Here, we launched the signal from input port A of both sides at the same time. When entering the MMIWA, the fundamental mode in the input single-lateralmode waveguide was expanded into multimodes. To simplify the problem, we considered the expansion of two modes that contained at least $90 \%$ of the total optical power. After propagating through the MMIWA, the optical signal entered the single-mode waveguide of the loop region where the field was amplified from port $\mathrm{C}$ to port $\mathrm{D}$. In the loop, the optical signal shared gain with the counterpropagating signal, which came from port D. From port D to port B in the MMIWA, there were also counterpropagating signals. The output power was extracted from port B.

For CW counterpropagation in the MMIWA, the TEpolarized electric field is expanded into two waveguide modes (modes 1 and 2), each of which propagates in both the $+z$ and $-z$ directions. Under the paraxial approximation, the four complex amplitudes $A_{1}^{+}(z), A_{1}^{-}(z), A_{2}^{+}(z)$, and $A_{2}^{-}(z)$ are slowly varying functions of $z$ and satisfy the following equations:

$$
\begin{aligned}
\frac{\partial A_{1}^{+}(z)}{\partial z}= & \frac{1}{2}(1+j \alpha) \overline{g_{1}}(z) A_{1}^{+}(z)+\frac{1}{2}(1+j \alpha) \overline{g_{x}}(z) \\
& \cdot A_{2}^{+}(z) e^{j \Delta \beta z}-\frac{1}{2} \rho A_{1}^{+}(z) \\
\frac{\partial A_{1}^{-}(z)}{\partial z}= & -\frac{1}{2}(1+j \alpha) \overline{g_{1}}(z) A_{1}^{-}(z)-\frac{1}{2}(1+j \alpha) \overline{g_{x}}(z) \\
& \cdot A_{2}^{-}(z) e^{-j \Delta \beta(z-L)}+\frac{1}{2} \rho A_{1}^{-}(z)
\end{aligned}
$$




$$
\begin{aligned}
\frac{\partial A_{2}^{+}(z)}{\partial z}= & \frac{1}{2}(1+j \alpha) \overline{g_{2}}(z) A_{2}^{+}(z)+\frac{1}{2}(1+j \alpha) \overline{g_{x}}(z) \\
& \cdot A_{1}^{+}(z) e^{-j \Delta \beta z}-\frac{1}{2} \rho A_{2}^{+}(z) \\
\frac{\partial A_{2}^{-}(z)}{\partial z}= & -\frac{1}{2}(1+j \alpha) \overline{g_{2}}(z) A_{2}^{-}(z)-\frac{1}{2}(1+j \alpha) \overline{g_{x}}(z) \\
& \cdot A_{1}^{-}(z) e^{j \Delta \beta(z-L)}+\frac{1}{2} \rho A_{2}^{-}(z)
\end{aligned}
$$

where

$$
\overline{g_{1(2)}}(z)=\int g(y, z)\left|F_{1(2)}(y)\right|^{2} d y
$$

and

$$
\overline{g_{x}}(z)=\int g(y, z) F_{1}(y) F_{2}(y) d y .
$$

Here, $g(y, z)$ is the gain constant distribution, $F_{1(2)}(y)$ is the normalized lateral distribution of waveguide modes 1 and $2, \rho$ is the linear loss constant, $\alpha$ is the linewidth enhancement factor, $\Delta \beta=\beta_{10}-\beta_{20}$ is the difference between the propagation constants of the two modes, and $L$ is the length of the MMIWA. Note that the variation along the epitaxial growth direction $(x)$ has been averaged out. Also, the gain constant distribution $g(y, z)$ is related to the optical intensity $|E(y, z)|^{2}$ through

$$
g(y, z)=\frac{g_{0}}{1+|E(y, z)|^{2} \tau_{c} \sigma / E_{\mathrm{sat}}}
$$

where $g_{0}$ is the small-signal gain constant, $\tau_{c}$ is the carrier lifetime, $\sigma$ is the effective cross-sectional area of the waveguide, and $E_{\text {sat }}$ is the saturation energy. The optical intensity can be explicitly expressed as

$$
\begin{aligned}
|E(y, z)|^{2}= & \frac{1}{2}\left[F_{1}^{2}(y)\left(\left|A_{1}^{+}(z)\right|^{2}+\left|A_{1}^{-}(z)\right|^{2}\right)\right. \\
& \left.+F_{2}^{2}(y)\left(\left|A_{2}^{+}(z)\right|^{2}+\left|A_{2}^{-}(z)\right|^{2}\right)\right] \\
& +F_{1}(y) F_{2}(y)\left|A_{1}^{+}(z)\right| \cdot\left|A_{2}^{+}(z)\right| \\
& \cdot \cos \left(-\Delta \beta \cdot z+\phi_{1}^{+}-\phi_{2}^{+}\right) \\
& +F_{1}(y) F_{2}(y)\left|A_{1}^{-}(z)\right| \cdot\left|A_{2}^{-}(z)\right| \\
& \cdot \cos \left(\Delta \beta \cdot(L-z)+\phi_{2}^{-}-\phi_{1}^{-}\right)
\end{aligned}
$$

where $\phi_{1(2)}^{+(-)}$are the phases of the complex amplitude $A_{1(2)}^{+(-)}$. Here we have neglected the fast-varying terms such as $\cos \left(2 \beta_{10(20)} z\right) \cos \left(\beta_{10} z+\beta_{20} z\right)$, etc. Note that, for counterpropagation in the single-mode loop waveguide, the equations above can also be used. However, they can be much simplified.

For numerical simulations, an iteration scheme was adopted to treat the counterpropagation problem. That is, at each iteration, the wave propagates in an environment determined by the counterpropagating of the last iteration. The iteration terminates when the steady state is attained, i.e., the optical fields change little from iteration to iteration. With typical parameter values in a GaAs-AlGaAs optical amplifier [15] and those of the experimental conditions, the simulation results, shown in Figs. 13 and 14, were obtained. For reasonable comparison with the corresponding results from experiments, the values of the vertical and horizontal axes represent the

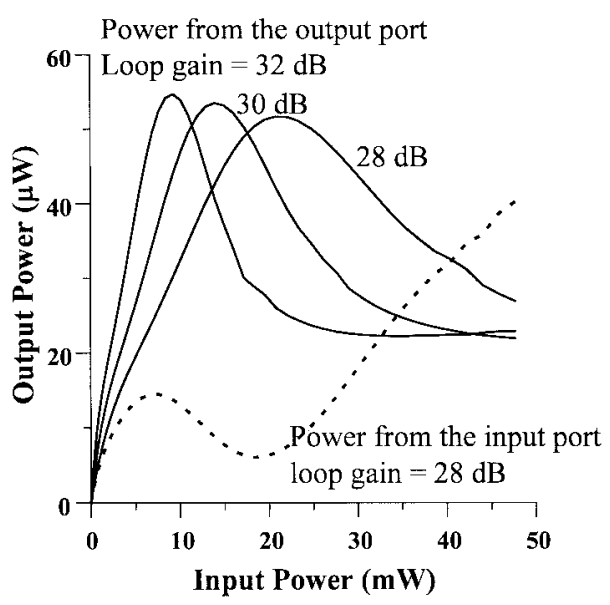

Fig. 13. Simulation results of the output power from the output port (solid curves) versus input power with three loop gain factors. The gain factor in the MMIWA is $22.5 \mathrm{~dB}$. The MMIWA and loop loss constants are set at 20 and $40 \mathrm{~cm}^{-1}$, respectively. The power emerging from the input port in the case of a $28-\mathrm{dB}$ loop gain factor is also presented with the dashed curve.

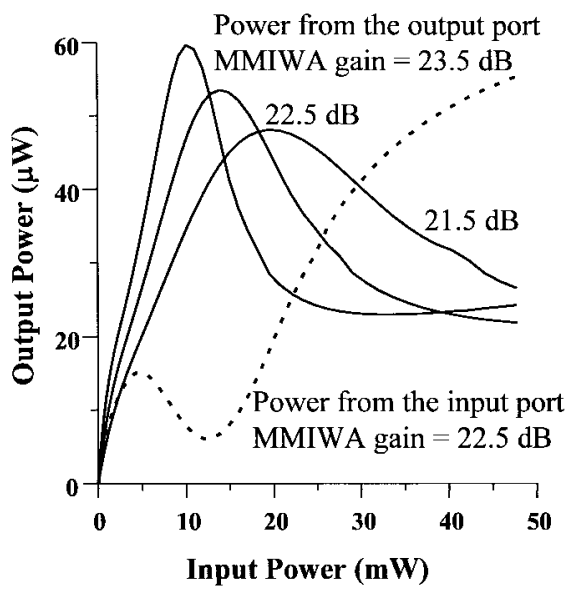

Fig. 14. Simulation results of the output power from the output port (solid curves) versus input power with three MMIWA gain factors. The loop gain factor is $30 \mathrm{~dB}$. The MMIWA and loop loss constants are set at 20 and 40 $\mathrm{cm}^{-1}$, respectively. The power emerging from the input port in the case of a 22.5-dB MMIWA gain factor is also presented with the dashed curve.

same output and input signal power levels in Figs. 3-11. In other words, the input power stands for that before entering the input port. In obtaining Figs. 13-18, we assumed that the input coupling efficiency was $1 \%$, and insufficient current injection onto the input-output region led to the reduction of signal power after propagating through either the input or output leg by $90 \%$. Note that we have also assumed the beat length of the MMIWA to be 0.375 . This choice was made simply for best fitting to the experimental data. These assumptions do not affect the demonstration of the general trends of device operation.

In Figs. 13 and 14, power-dependent switching results, just like those from experiments, can be seen. In Fig. 13, the smallsignal gain factor in the MMIWA was fixed at $22.5 \mathrm{~dB}$. The three solid curves represent the power from the output port in the cases of small-signal gain factors in the loop at 28, 30, and $32 \mathrm{~dB}$, respectively. In the experiment, the loop gain factor depends on the bending loss of the waveguide and the injection 


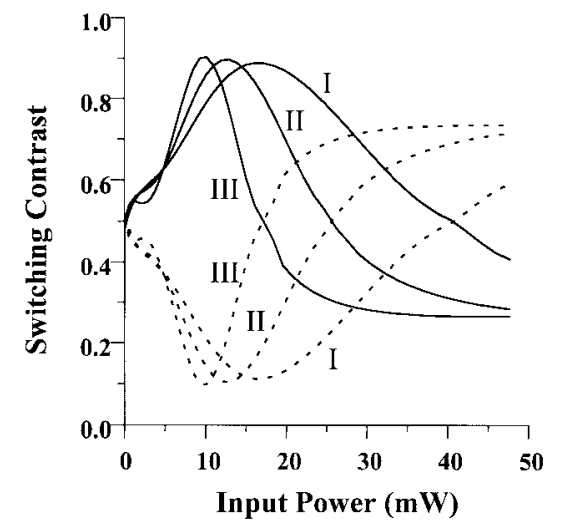

Fig. 15. Switching contrast ratios of three MMIWA gain factors corresponding to the cases of Fig. 14. Curves I, II, and III represent the MMIWA gain factors equal to $21.5,22.5$, and $23.5 \mathrm{~dB}$, respectively.

current $I_{3}+I_{4}$. Hence, Fig. 13 can be compared with Fig. 5 qualitatively. The right-shift trends of the output maximum with a decreasing loop gain factor and $I_{3}+I_{4}$ level coincide well. A similar comparison can be made between Figs. 14 and 4. In Fig. 14, the injection current $I_{2}$ onto the MMIWA is varied so that its gain factor changes from 21.5 to $23.5 \mathrm{~dB}$. Although the variation ranges of the injection current are different, the two figures show the same trend, i.e., the output maximum right-shifts with decreasing $I_{2}$. Experimentally, the output peak power drops with decreasing injection current onto either the loop or MMIWA. The same trend is observed in the simulation results.

Typically, both signals emerging from the output and input ports are useful in a conventional NOLM device. However, because an antireflection coating was not prepared for our device, it is difficult to measure the signal power emerging from the input port. The dashed curves in Figs. 13 and 14 show the corresponding signal power emerging from the input port when the loop gain factor is $28 \mathrm{~dB}$ in Fig. 13 and the MMIWA gain factor is $22.5 \mathrm{~dB}$ in Fig. 14. From these results, one can see that the signal power levels from the input and output ports are mutually complementary. In either Figs. 13 or 14 , for illustration clarity, only one dashed curve was plotted. The results corresponding to the other two solid curves are similar. Having the simulated results of the power emerging from the output and input ports, we can evaluate the switching ratio of our device. Fig. 15 shows the switching contrast ratios as functions of the input power for three MMIWA gain factors, corresponding to the cases in Fig. 14. We can see that the best switching contrast variation is from $0.9 / 0.1$ to $0.26 / 0.74$ by varying the input power from 10 to $35 \mathrm{~mW}$ (curve III).

As discussed earlier, the operation principle of our device is quite different from that of a conventional NOLM. In our device, nonlinear coupling in the MMIWA plays a crucial role. To demonstrate the nonlinear coupling effect in the MMIWA, we show the evolution of the lateral field distribution after propagating through an isolated MMIWA. Fig. 16(a) and (b) show the output lateral intensity and phase distributions, respectively, of two input power levels. For comparison, the input intensity and phase distributions are depicted with the dotted curves. The propagation results of the low $\left(10^{-18} \mathrm{~mW}\right)$

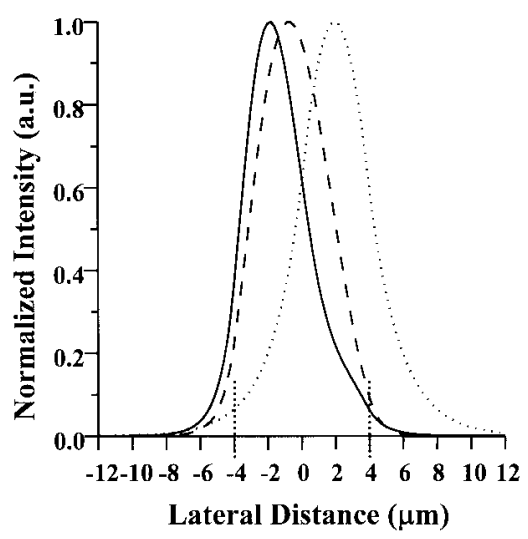

(a)

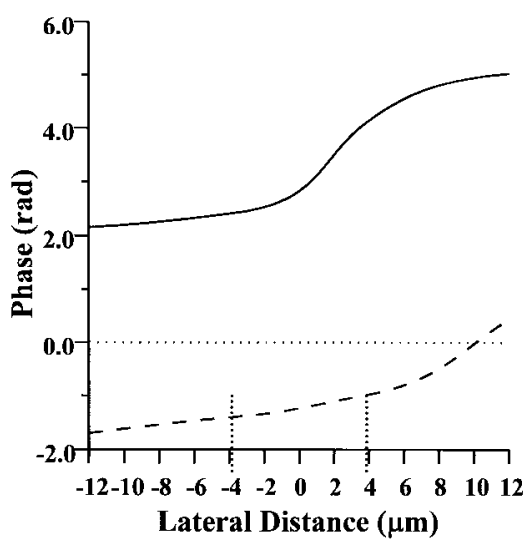

(b)

Fig. 16. (a) Output lateral intensity and (b) phase distributions of an isolated MMIWA with two input power levels: $10^{-18}$ (solid curves) and $25 \mathrm{~mW}$ (dashed curves). The input signal is depicted with the dotted curves. The two vertical dotted lines show the region of waveguide ridge loading.

and high $(25 \mathrm{~mW})$ input power cases are plotted with the solid and dashed curves, respectively. Note that input power values here represent those at the input plane of the MMIWA. The MMIWA parameter values are the same as those for Fig. 14 with the gain factor equal to $22.5 \mathrm{~dB}$. It can seen that with the MMIWA length equal to 0.375 beat length, the major part of the signal power emerges from the left-hand side in the lateral dimension. However, the high input power leads to nonlinear coupling and, hence, more power staying on the right-hand side. Besides the change of intensity distribution, the phase varies with input power level. It is noted that, when the gain factor is varied, the results of the low-power case are not significantly changed. Nevertheless, those of the high-power case become quite different because of different gain and gain saturation effects. With the results shown in Fig. 16(a) and (b), we evaluated the power ratio and relative phase of the two components coupled into the two ends of the loop structure. It was found that the power splitting ratio (cross port/bar port) was $5.22(1.59)$ and the relative phase was $1.51(0.38)$ in the low (high) input power case. From those values, one can again see the strong effect of nonlinear coupling in the MMIWA. The results of the low input power case are not much different from those of a directional coupler of the same length, i.e., 0.375 beat length $(5.76$ for the splitting ratio and $\pi / 2$ for 


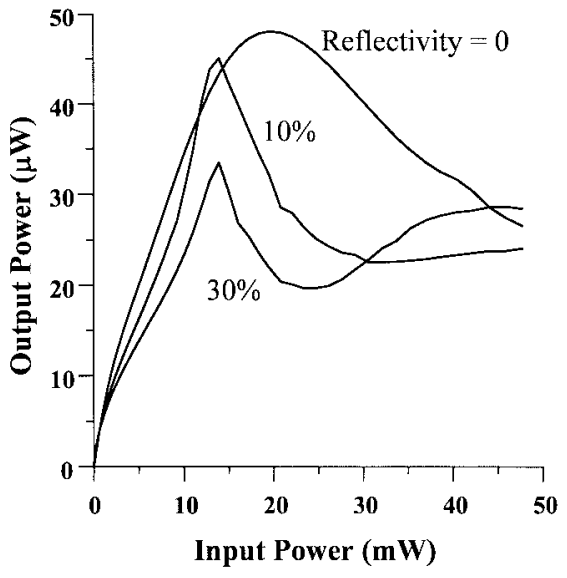

Fig. 17. Simulation results of the output power versus input power with three end-facet reflectivity values. The loop gain factor is $30 \mathrm{~dB}$. The MMIWA gain factor is $21.5 \mathrm{~dB}$. The MMIWA and loop loss constants are set at 20 and 40 $\mathrm{cm}^{-1}$, respectively.

the relative phase). With a $3-\mathrm{dB}$ directional coupler $(0.25$ beat length), the splitting ratio is 1 , which guarantees total reflection of weak signals in a conventional NOLM. However, the designated unequal power splitting of the MMIWA leads to almost equal reflected and transmitted powers when a weak signal is applied to our nonlinear loop device. This difference can be easily understood by realizing that in the round trip the signal experiences coupling of in total one-half beat length in a conventional NOLM with a $3-\mathrm{dB}$ coupler. With the loop structure for exchanging the bar- and cross-signals, the lowpower signal is expected to be totally reflected. Nevertheless, in our device, the total coupler length in the round trip is a beat length of 0.75 , which leads to equal reflection and transmission (see Fig. 15). Note that, in the operation of our device, there exist counterpropagating signals in the MMIWA. Between the two counterpropagating signals, the feedback signal from the loop structure is much stronger than that of the other direction. The interplay between the two counter-propagating signals determines the nonlinear coupling effects in the MMIWA, which are more complicated than shown in Fig. 16(a) and (b). This interplay is dominated by the feedback signal.

Without an antireflection coating, the out-coming signal from either the input or output port is partially reflected at the end facet. Normally, the reflectivity can be as high as $30 \%$. To elucidate the effects of the end-facet reflection, in Fig. 17 we plot three curves of different end-facet reflectivity values corresponding to the operation conditions of the $21.5-\mathrm{dB}$ MMIWA gain factor case in Fig. 14. One can see that the endfacet reflection sharpens the output peak (from the output port) and effectively reduces the switching power. It was estimated that the end-facet reflectivity in our device was between $10 \%$ and $30 \%$. The mechanisms of the reflection effects on device operation deserve further investigation.

To confirm the important function of the loop structure, as shown experimentally, we also conducted numerical simulations for comparing the loop and the mirror cases in Fig. 11. Fig. 18 illustrates the simulation results for the four cases: loop with gain (curve I, normal function of the device with the loop gain factor at $30 \mathrm{~dB}$ ), loop without gain (curve

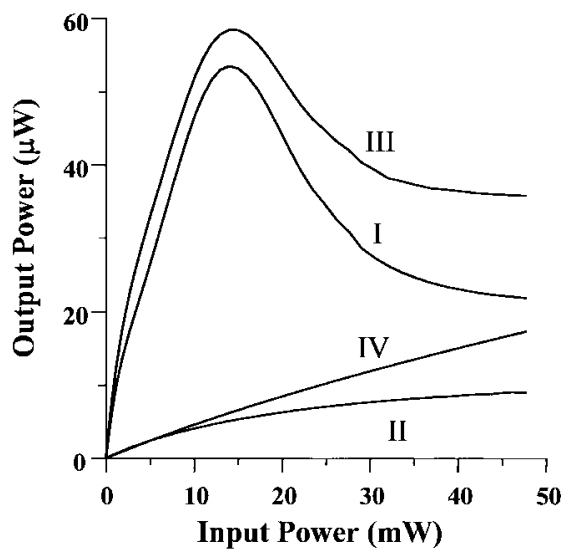

Fig. 18. Simulation results for four cases: loop with gain (I), loop without gain (II), mirror with gain (III), and mirror without gain (IV). The MMIWA gain factor is always $22.5 \mathrm{~dB}$. The loop gain factor, whenever available, is set at $30 \mathrm{~dB}$. The MMIWA and loop loss constants are set at 20 and $40 \mathrm{~cm}^{-1}$, respectively.

II, corresponding to the case of a small $I_{3}+I_{4}$ value), mirror with gain (curve III, loop removed with imaginary signal amplification of the loop gain factor at $30 \mathrm{~dB}$ ), and mirror without gain (curve IV, loop removed). In all curves, the MMIWA gain factor is set at $22.5 \mathrm{~dB}$. One can see that, when the loop gain is zero (curves II and IV), no significant power-dependent switching behaviors are observed. The clear nonlinear switching in curves I and III indicates the importance of signal amplification in the loop. Note that in device operation curve I is different from III by the MMIWA lateral field redistribution through the loop structure. The more efficient nonlinear switching result in curve I indicates the importance of the field redistribution function of the loop. This conclusion is consistent with that of the experiments, as shown in Fig. 11.

\section{CONCLUSION}

We have fabricated an all-semiconductor optical-amplifier based Sagnac interferometer device and characterized its operation with $\mathrm{CW}$ optical signals. The operational principle of the device was quite different from that of a conventional NOLM. In our device, gain saturation in the MMIWA led to a nonlinear coupling phenomenon. When this phenomenon combined with signal amplification and lateral field redistribution from the loop, efficient nonlinear switching occurred. Power-dependent self-switching and cross-switching behaviors were studied with signals of different wavelengths and polarization. Experimental results revealed that the operation of the all-active device was strongly affected by the optical gain levels in different portions of the device. A theoretical model was established to numerically simulate the device operation. The numerical results agreed very well in trend with the experimental data. They also elucidated the importance of the loop structure for efficient device operation. The effects of the end-facet reflection were also discussed. Further studies include the implementation and theoretical understanding of device operation with pulsed signals. With pulsed signals, the asymmetry between the two counterpropagating signals 
in the loop structure becomes important. Meanwhile, for fiber communication applications, similar devices for operation near $1550 \mathrm{~nm}$ will be implemented.

\section{REFERENCES}

[1] C. Kolleck and U. Hempelmann, "All-optical wavelength conversion of NRZ and RZ signals using a nonlinear optical loop mirror," J. Lightwave Technol., vol. 15, pp. 1906-1913, 1997.

[2] I. D. Phillips, A. Gloag, P. N. Kean, N. J. Doran, I. Bennion, and A. D. Ellis, "Simultaneous demultiplexing, data regeneration, and clock recovery with a single semiconductor optical amplifier-based nonlinearoptical loop mirror," Opt. Lett., vol. 22, pp. 1326-1328, 1997.

[3] X. Chen, B. Hoanca, K. Feng, J. Cai, and A. E. Willner, "Experimental demonstration of fast simultaneous wavelength switching and time demultiplexing using a nonlinear optical loop mirror," IEEE Photon. Technol. Lett., vol. 9, pp. 919-921, 1997.

[4] N. J. Doran and D. Wood, "Nonlinear-optical loop mirror," Opt. Lett., vol. 13 , pp. 56-58, 1988.

[5] J. Cai, K. Feng, X. P. Chen, and A. E. Willner, "Equalization of nonuniform EDFA gain using a fiber-loop mirror," IEEE Photon. Technol. Lett., vol. 9, pp. 916-918, 1997.

[6] M. E. Fermann, F. Haberl, M. Hofer, and H. Hochreiter, "Nonlinear amplifying loop mirror," Opt. Lett., vol. 15, pp. 752-754, 1990.

[7] I. N. Duling, III, C.-J. Chen, P. K. Wai, and C. R. Menyuk, "Operation of a nonlinear loop mirror in a laser cavity," IEEE J. Quantum Electron., vol. 30, pp. 194-199, 1994

[8] M. Eiselt, W. Pieper, and H. G. Weber, "SLALOM: Semiconductor laser amplifier in a loop mirror," J. Lightwave Technol., vol. 13, pp. 2099-2112, 1995.

[9] J. P. Sokoloff, P. R. Prucnal, I. Glesk, and M. Kane, "A terahertz optical asymmetric demultiplexer," IEEE Photon. Technol. Lett., vol. 5, pp. 787-790, 1993.

[10] E. Jahn, N. Agrawal, W. Pieper, H.-J. Ehrke, D. Franke, W. Furst, and C. M. Weinert, "Monolithically integrated nonlinear Sagnac interferometer and its application as a $20 \mathrm{Gbit} / \mathrm{s}$ all-optical demultiplexer," Electron. Lett., vol. 32, pp. 782-784, 1996.

[11] M. C. Shih, M. B. Freier, G. Haase, R. Scarmozzino, and R. M. Osgood, Jr., "Condensed chlorine etching of GaAs induced by excimer laser radiation," Appl. Phys. Lett., vol. 61, pp. 828-830, 1992.

[12] M. C. Shih, M. B. Freier, R. Scarmozzino, and R. M. Osgood, Jr., "Patterned, photon-driven cryoetching of GaAs and AlGaAs," J. Vac. Sci. Technol., vol. B13, pp. 43-54, 1995.
[13] C.-H. Tsai, J.-H. Lee, H.-J. Chiang, C. C. Yang, M. C. Shih, B. C. Chen, T. J. Chuang, and Y. Chang, "Semiconductor circular ring lasers fabricated with the cryo-etching technique," IEEE Photon. Technol. Lett., vol. 10, pp. 751-753, 1998 .

[14] P. LiKamWa, A. Miller, C. B. Park, J. S. Roberts, and P. N. Robson, "All optical switching of picosecond pulses in GaAs quantum well waveguide coupler," Appl. Phys. Lett., vol. 57, pp. 1846-1849, 1990.

[15] L. A. Coldren and S. W. Corzine, Diode Lasers and Photonic Integrated Circuits. New York: Wiley, 1995.

Jiun-Haw Lee, photograph and biography not available at the time of publication.

Ding-An Wang, photograph and biography not available at the time of publication.

Yean-Woei Kiang, photograph and biography not available at the time of publication.

Hsin-Jiun Chiang, photograph and biography not available at the time of publication.

Ding-Wei Huang, photograph and biography not available at the time of publication.

C. C. Yang (S'81-M'83-SM'93), photograph and biography not available at the time of publication. 